\title{
Modal Test Optimization Using VETO (Virtual Environment for Test Optimization)
}

Scott E. Klenke, Garth M. Reese, Larry A. Schoof, Craig Shierling

$$
\text { () S P }
$$

Prepared by

Sandia National Laboratories

Albuquerque, New Mexico 87185 and Livermore, California 94550

for the United States Department of Energy

under Contract DE-AC04-94AL85000

sixing

Approved for public release; distribution is unlimited.
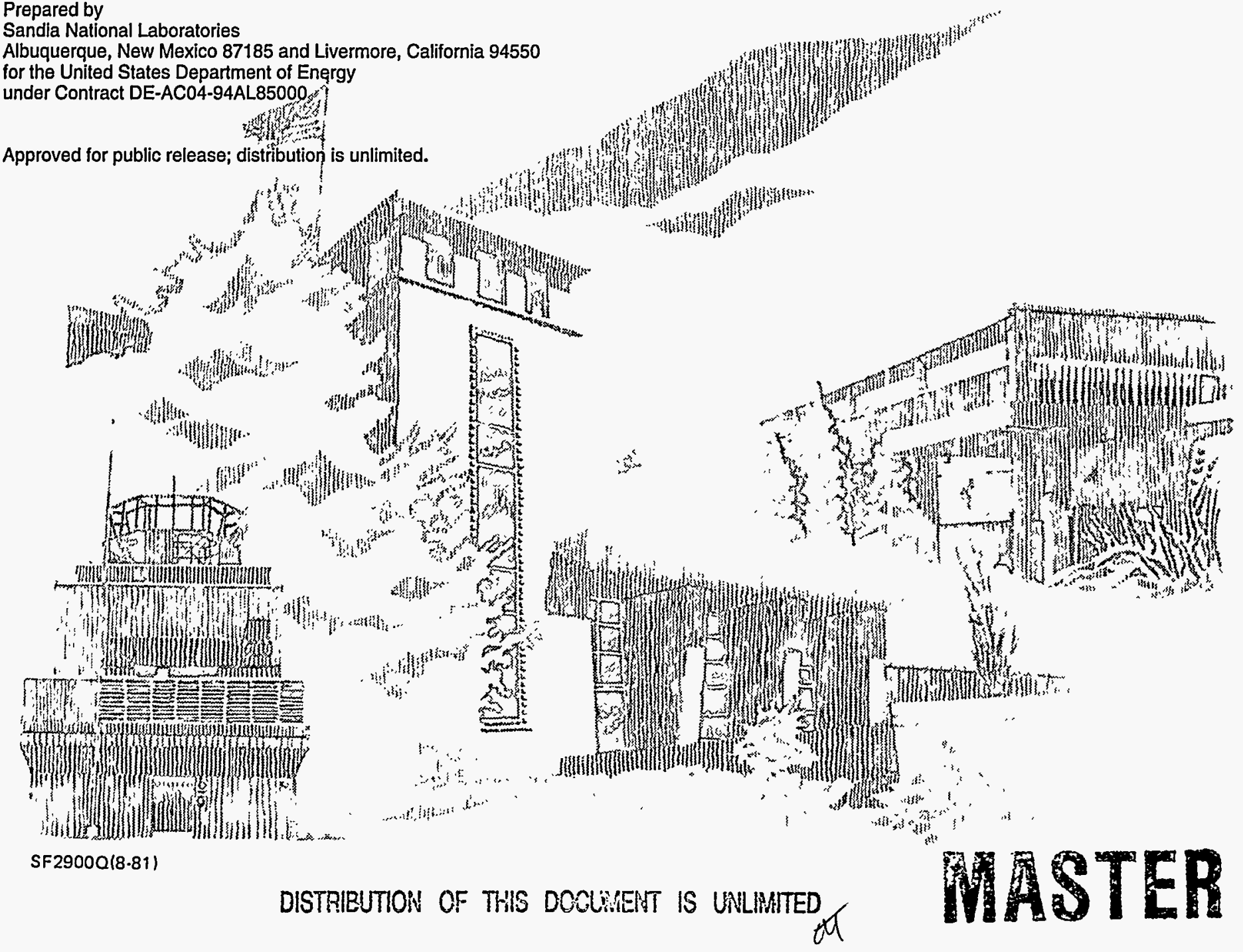
Issued by Sandia National Laboratories, operated for the United States Department of Energy by Sandia Corporation.

NOTICE: This report was prepared as an account of work sponsored by an agency of the United States Government. Neither the United States Government nor any agency thereof, nor any of their employees, nor any of their contractors, subcontractors, or their employees, makes any warranty, express or implied, or assumes any legal liability or responsibility for the accuracy, completeness, or usefulness of any information, apparatus, product, or process disclosed, or represents that its use would not infringe privately owned rights. Reference herein to any specific commercial product, process, or service by trade name, trademark, manufacturer, or otherwise, does not necessarily constitute or imply its endorsement, recommendation, or favoring by the United States Government, any agency thereof or any of their contractors or subcontractors. The views and opinions expressed herein do not necessarily state or reflect those of the United States Government, any agency thereof or any of their contractors.

Printed in the United States of America. This report has been reproduced directly from the best available copy.

Available to DOE and DOE contractors from Office of Scientific and Technical Information PO Box 62

Oak Ridge, TN 37831

Prices available from (615) 576-8401, FTS 626-8401

Available to the public from National Technical Information Service

US Department of Commerce

5285 Port Royal Rd

Springfield, VA 22161

NTIS price codes

Printed copy: A03

Microfiche copy: A01 


\title{
Modal Test Optimization Using VETO (Virtual Environment for Test Optimization)
}

\author{
Scott E. Klenke, Garth M. Reese, \\ Larry A. Schoof and Craig Shierling (RE/SPEC) \\ Sandia National Laboratories \\ Albuquerque, New Mexico
}

\begin{abstract}
We present a software environment integrating analysis and test-based models to support optimal modal test design through a Virtual Environment for Test Optimization (VETO). A goal in developing this software tool is to provide test and analysis organizations with a capability of mathematically simulating the complete test environment in software. Derived models of test equipment, instrumentation and hardware can be combined within the VETO to provide the user with a unique analysis and visualization capability to evaluate new and existing test methods. The VETO assists analysis and test engineers in maximizing the value of each modal test. It is particularly advantageous for structural dynamics model reconciliation applications.

The VETO enables an engineer to interact with a finite element model of a test object to optimally place sensors and exciters and to investigate the selection of data acquisition parameters needed to conduct a complete modal survey. Additionally, the user can evaluate the use of different types of instrumentation such as filters, amplifiers and transducers for which models are available in the VETO. The dynamic response of most of the virtual instruments (including the device under test) is modeled in the state space domain. Design of modal excitation levels and appropriate test instrumentation are facilitated by the VETO's ability to simulate such features as unmeasured external inputs, A/D quantization effects, and electronic noise. Measures of the quality of the experimental design, including the Modal Assurance Criterion, and the Normal Mode Indicator Function are available. The VETO also integrates tools such as Effective Independence and minamac to assist in selection of
\end{abstract}


optimal sensor locations. The software is designed about three distinct modules:

1. a main controller and graphical user interface (GUI) written in $\mathrm{C}++$,

2. a visualization model, taken from FEAVR, running under $\mathrm{AVS},{ }^{\dagger}$ and

3. a state space model and time integration module, built in SIMULINK.

These modules are designed to run as separate processes on interconnected machines. MATLAB's external interface library is used to provide transparent, bidirectional communication between the controlling program and the computational engine where all the time integration is performed. Data from the finite element model is downloaded to the MATLAB engine where the SIMULINK model is automatically created and executed. MATLAB GUI elements are used to simulate the data acquisition environment including response traces, over-range indicators, and full-scale voltage ranges.

${ }^{\dagger}$ AVS is a trademark of Advanced Visual Systems, Inc., Waltham, MA.

${ }^{\ddagger}$ MATLAB and SIMULINK are trademarks of The Math Works, Inc., Natick, MA. 


\section{Contents}

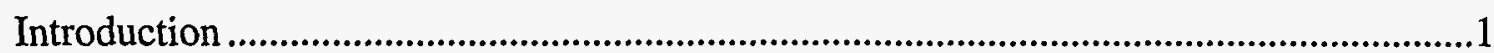

Program Integration - Unix ${ }^{\mathrm{TM}}$ Socket Connected Modules..................................................

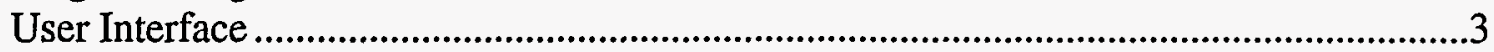

Visualization with AVS Finite Element Analysis VieweR (FEAVR) .................................6

Simulations with MATLAB and SIMULINK ...............................................................

Application of the VETO to Structural Dynamics Test Simulation ...................................12

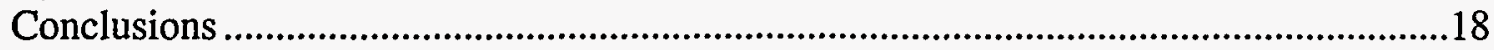

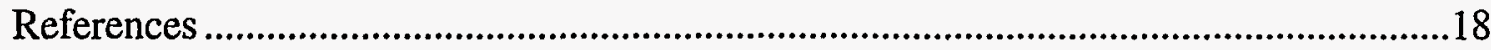

\section{Figures}

1. VETO Main User Interface ......................................................................................

2. Virtual Instrument Control Panel for Sensors...........................................................4

3. Instrument Input Panel for Sensors .......................................................................

4. VETO Front End Sampling Panel ...................................................................................5

5. VETO Front End Measurement Panel ........................................................................

6. FEAVR Display .................................................................................................

7. Deformed FE Model and Trace Lines From FEAVR .....................................................

8. Partial Front End Block Diagram ............................................................................10

9. Simulation Monitor in Its Initial State ...................................................................11

10. VETO Test Design for the Housing ................................................................13

11. Estimated Frequency Shifts Due to Mass Loading the Housing...................................13

12. Normal Mode Indicator Function for the Housing........................................................14

13. Driving Point Frequency Response for Housing...........................................................14

14. Modal Assurance Criterion (MAC) of the Housing .......................................................15

15. Partial Block Model of SIMULINK Environment.................................................16

16. Collected Responses From Simulation Monitor ......................................................16

17. Comparison of Simulated and Experimental Frequency Response Functions ...........17

\section{Table}

1. Data Requirements in Each Environment ............................................................... 
Intentionally Left Blank

$-\mathrm{iv}-$ 


\section{Modal Test Optimization Using VETO (Virtual Environment for Test Optimization)}

\section{Introduction}

This paper presents an innovative test/analysis tool, called the Virtual Environment for Test Optimization (VETO), which reduces test instrumentation iteration and thereby produces better modal tests. Communication between test and analysis engineers is enhanced early in the design cycle. Traditionally, the role of testing in the product realization process is limited to the end of the design cycle, after hardware has already been produced. As a result, data analysis and test requirements for a component are only considered when the hardware is scheduled for testing. Thus, the full benefit of the analysis in guiding the test is not realized. A goal in developing this software tool is to provide test and analysis organizations with a capability of mathematically simulating the complete test environment in software. Derived models of test equipment, instrumentation and hardware can be combined within the VETO to provide the user with a unique analysis and visualization capability to evaluate new and existing test methods. By providing engineers with a tool that allows them to optimize an experimental design within a computer environment, pretest analysis can be performed using analytical models to rapidly evaluate components before manufacturing. The benefits of using this type of experimental design tool can be very extensive. The user can evaluate the use of different types of test instrumentation and equipment as well as investigate new testing techniques for system identification to be used in analysis/experimental model validation.

The VETO also plays a crucial role in support of an ongoing Sandia National Laboratories program called Knowledge Based Testing. The Knowledge Based Testing program incorporates aspects of design, analysis, and test with rapid prototyping to optimize test-based product information from an experiment. Combining the use of the VETO (to optimally design an experiment) with the use of rapid prototyping techniques for generating component parts makes the processes of model updating and validation much more efficient. There is a critical need for providing test-based information to produce confidence in the predictive capabilities of computational models. A test design tool such as the VETO can make a large impact in addressing this need.

The initial step in the Knowledge Based Testing program is generation of a computer aided design model which represents the geometry of the component to be tested. This geometric model is then used to assist component visualization, to generate a computational model used for dynamic analysis, and to produce a rapid prototype component through a stereolithography or "fastcast" process. ${ }^{\dagger}$ The developed computational model of the component is combined with analytical and/or experimentally derived models of the test instrumentation within the VETO to determine an optimal test

\footnotetext{
†Fastcast uses a stereolithography part as a mold for an automatic metal casting system.
} 
design. The VETO is used to simulate the experiment and to maximize the test-based information for computational model validation. The next step in the Knowledge Based Testing program is performance of the experimental test on the rapid prototype component based on the VETO test design. The results of this experiment are then used to validate the computational model.

A major objective of this software development effort is flexibility. Because the virtual environment is a prototype software system, a primary concern for its design is to make the code easy to develop. To minimize our effort, existing software tools are used wherever possible, provided that the necessary functionality and flexibility are available. Another significant design objective is to provide a final software system that can be used by a variety of individuals who have not been involved in its development. As described further in this report, our design integrates several commercial tools to meet these objectives.

The major tasks involved in our effort include: 1) database management, 2) visualization of the device under test, 3 ) utility functions (such as those providing additional information about any of the instruments, or interconnecting them), and 4) time integration of the system. A key element within the VETO environment is the use of virtual instruments to simulate dynamic behavior of real instruments. Each virtual instrument may require a different data representation within the different VETO modules. For example, the device under test requires a geometric definition under the visualization module, while the model is reduced to state space ABCD matrices for use in the time integration module. The required data for the different analysis environments are shown in Table 1.

Table 1. Data Requirements in Each Environment

\begin{tabular}{|l|l|l|l|}
\hline \multirow{2}{*}{ Virtual Instrument } & \multicolumn{3}{c|}{ Environment } \\
\cline { 2 - 4 } & visualization & database & time integration \\
\hline \hline Device Under Test (DUT) & $\begin{array}{l}\text { geometry } \\
\text { eigenvectors } \\
\text { eigenvalues }\end{array}$ & $\begin{array}{l}\text { eigenvectors } \\
\text { eigenvalues }\end{array}$ & ABCD matrices \\
\hline sensors \& actuators & $\begin{array}{l}\text { grid number } \\
\text { orientation }\end{array}$ & $\begin{array}{l}\text { connections } \\
\text { state model name } \\
\text { parameters }\end{array}$ & $\begin{array}{l}\text { connections } \\
\text { state model name } \\
\text { parameters }\end{array}$ \\
\hline filters \& amplifiers & N/A & $\begin{array}{l}\text { connections } \\
\text { state model name } \\
\text { parameters }\end{array}$ & $\begin{array}{l}\text { connections } \\
\text { state model name } \\
\text { parameters }\end{array}$ \\
\hline Front End system & N/A & N/A & $\begin{array}{l}\text { construction } \\
\text { based on other } \\
\text { instruments }\end{array}$ \\
\hline
\end{tabular}




\section{Program Integration - Unix ${ }^{\mathrm{TM}}$ Socket Connected Modules}

The VETO software is divided into three main modules: 1) user interface, database and utilities, 2) visualization, and 3) time integration. The user interface and utilities are written in $\mathrm{C}++$ with the database implemented using the netCDF library. ${ }^{\dagger}$ Visualization is performed using previously developed, custom AVS (Advanced Visual Systems) networks [1]. Use of existing visualization software immediately made available a wealth of tools permitting visualization of extensive finite element results, including modes of vibration, strain energy densities and static response data. The time integration module uses MATLAB and its SIMULINK toolkit. MATLAB was also used to construct the state space models for many of the virtual instruments (such as amplifiers and filters).

Communication between these different modules is performed using unix sockets. MATLAB is released with a set of interprocess communication tools (the "external interface"), by which data may be easily transferred between the programs. Data transfer with AVS was more complicated but accomplished in a similar fashion. AVS is distributed with example code permitting execution in a "server" mode. Commands and parameters may be readily transferred to AVS, but only the results printed to standard output may be retrieved; there is no direct access to internal data structures. This is a significant limitation of the software. Most of the structural data were shared through EXODUS II [2] files used by the AVS visualization software. The random-access data features of this format were quite important for implementation of many of the analysis tools discussed later in the report.

In addition to permitting rapid implementation of the virtual environment concepts, separation of the VETO software into these three modules had important side benefits. Unix sockets are network transparent, permitting us to run the application segments on different machines. For example, AVS could be run on an SGI (Silicon Graphics, Inc.) machine specifically designed for visualization problems, while MATLAB ran on a more generalpurpose computer platform. Program development and debugging were also facilitated by the complete separation of these processes.

\section{User Interface}

Database, integration, utility and user interface functions are performed in the vetomain module (see Figure 1). The "File" option of the vetomain menu bar allows users to load finite element (FE) models and previously defined virtual test files into the VETO software. This module also provides numerous tools to assist the engineer in understanding how the various virtual instruments interact together.

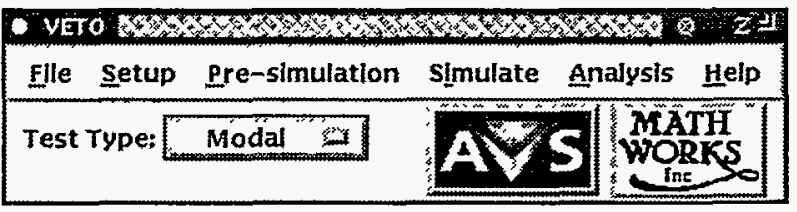

Figure 1. VETO Main User Interface

${ }^{\dagger}$ netCDF is a public domain, machine-independent data format available from the Unidata Program Center in Boulder (unidata.ucar.edu). 
The user interface is implemented in $\mathrm{C}$ and $\mathrm{C}++$ using Motif libraries. To provide access to AVS and MATLAB, user interface widgets were constructed by which commands could be entered and all output from the applications displayed. Vetomain acts as a controller, sending and retrieving data from the other two applications. Some limitations in the event-driven model were introduced by the separation of the three application codes. Each module has its own event loop, specific to the interface events of that application. However, some events, such as communicating the node numbers from the visualized model, would be more natural if clicking on the model directly communicated back to the database program. The single socket connection between programs makes this quite awkward. Action-specific code was written to deal with node numbering; however, a more elegant solution might utilize an additional communication channel along with integrated event loops.

Vetomain is used to construct parametric models of the instruments and to formulate interconnections between the models. Each of the virtual instruments is constructed in customized control panels. The user is able to interact with the Virtual Instruments Control Panel, selected from the "Setup/Instruments" option, to provide and view information on the devices in the simulation (see Figure 2). A typical control panel used in the design of the virtual actuators and sensors, which are to be in contact with the device under test (DUT), is shown in Figure 3.

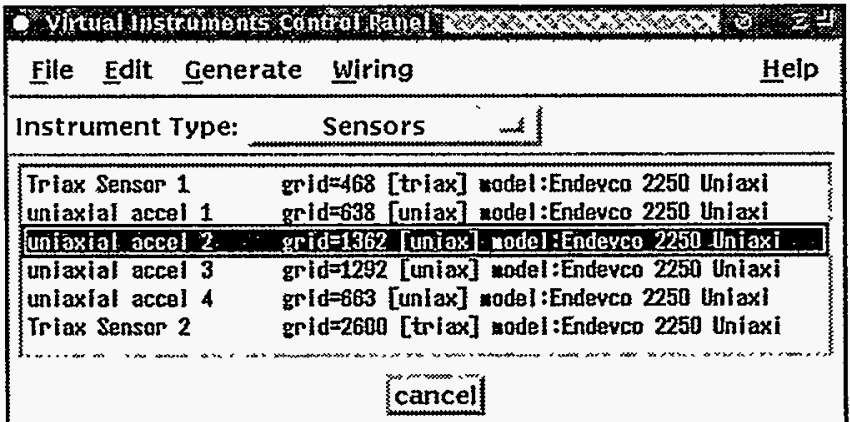

Figure 2. Virtual Instrument Control Panel for Sensors

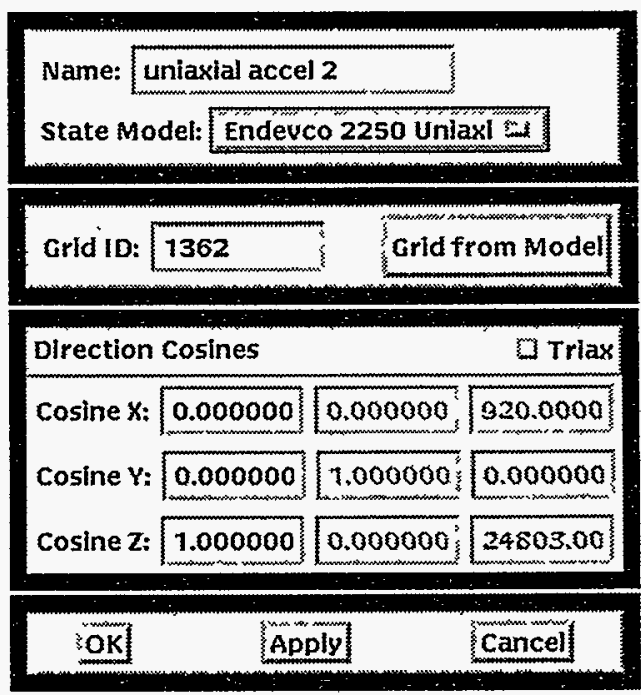

Figure 3. Instrument Input Panel for Sensors 
Before initiating a simulation run, the user also needs to define additional instruments, such as filters, amplifiers and the Front End data acquisition device. Two subpanes for providing parameters needed for sampling and computation of post-simulation analysis measurements for the Front End model are shown in Figures 4 and 5. There are additional subpanes to specify triggering, auto-ranging, windowing, averaging and display parameters for the simulation of the Front End device. A special instrument called a "wire" is used to connect multiple instruments together. Once assembled, the DUT, selected virtual instruments and wire connections form the virtual test environment.

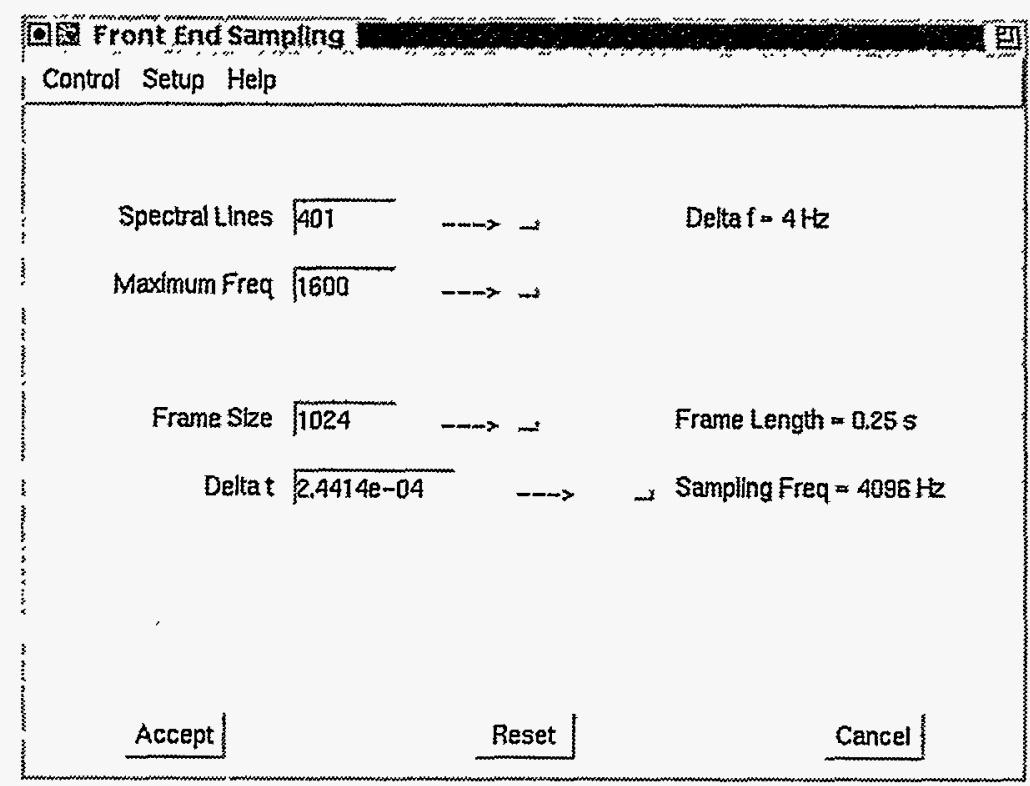

Figure 4. VETO Front End Sampling Panel

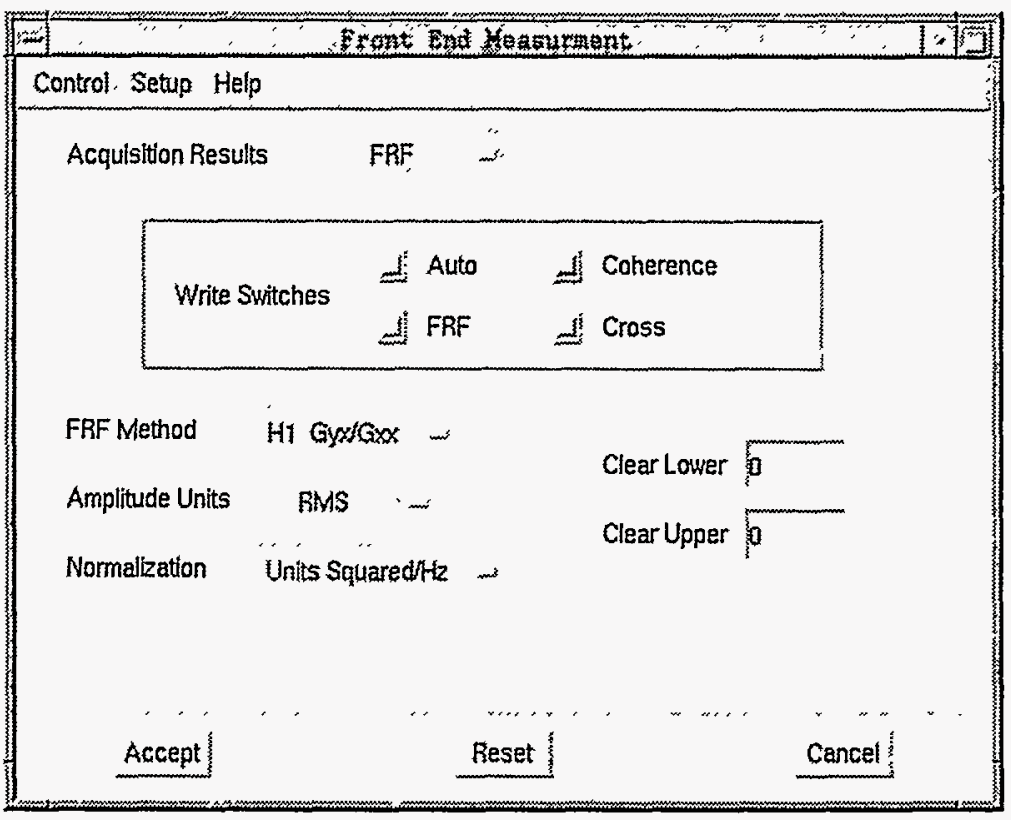

Figure 5. VETO Front End Measurement Panel 
Data defining the virtual instruments and the structure of the test environment are downloaded to MATLAB, the state space model of the structure or device under test is constructed, and the ABCD matrices are stored in the MATLAB workspace. SIMULINK scripts are then called to organize the virtual instrument models and data into an integration network from which the time histories of the system are computed.

The vetomain module also provides numerous evaluation tools to allow the test engineer to determine the completeness of the virtual test environment. These tools can be accessed through the "Pre-simulation" menu option from vetomain. The modal data used for visualization is combined with selected virtual instruments to compute the Modal Assurance Criterion (MAC) [3], ${ }^{\dagger}$ normal mode indicator functions [3], and driving point frequency response. The effects of the mass loading of the structure by the sensors may also be computed using a perturbation method. These and other tools guide the engineer in the design of tests that will accurately identify all the desired modes of the structure.

Even with these tools, placement of sensors can be a difficult task. Tools such as the effective independence [4] method or the minamac [3] are used to automatically place sensors in locations which may help optimize the information available from the test. The virtual test environment provides the engineer with immediate visual feedback to determine the success of these methods, for which some engineering interaction is still required. Methods for automatic selection of actuator locations are currently under consideration.

\section{Visualization With AVS Finite Element Analysis VieweR (FEAVR)}

Since an FE analysis is typically performed to predict the modes of vibration of a device, it was decided to utilize the FE model as the primary geometric representation of the device for visualization purposes within VETO. A prototype environment, FEAVR [1], which had been developed to provide a general purpose visualization capability for $\mathrm{FE}$ analyses, was selected as the graphics tool. FEAVR is an interface to the broad FE visualization functionality of AVS, incorporating networks and modules written or customized at Sandia. By using FEAVR, a user is freed from knowing the details of AVS. As an FE analysis visualization system, FEAVR provides the following capabilities:

- colors the model with color fringes representing element-based (e.g., stress, strain, etc.) or node-based (e.g., temperature, displacement) values.

- slices the model by showing an interior cutting plane or by removing a portion of the model that lies on one side of a slice plane.

- creates an isosurface which is a surface on which an element-based or node-based scalar value is constant.

- represents a vector field (velocity, for instance) as arrows or streamlines (continuous lines that are everywhere tangent to the vector field).

${ }^{\dagger}$ The MAC is a normalized measure of mode orthogonality. It is defined by,

$$
\operatorname{MAC}_{i j}=\frac{\left(\varphi_{i}^{T} \cdot \varphi_{j}\right)^{2}}{\left(\varphi_{i}^{\mathrm{T}} \cdot \varphi_{i}\right)\left(\varphi_{j}^{\mathrm{T}} \cdot \varphi_{j}\right)}
$$

where $\varphi_{j}$ is the eigenvector of the $j^{\text {th }}$ mode. The MAC is most often used to determine correspondence between test and analysis. In this effort, it identifies completeness of the modes. The MAC is sampled only at sensor locations, hence an incomplete sensor set results in large off-diagonal terms. 
- creates $X-Y$ plots of variables as they vary through time or distance (across or through the model).

- deforms the model according to a vector field, typically a displacement vector.

- creates animations of mode shapes.

- "probes" the model to determine mesh-related values (i.e., nodal coordinates, node ID, element ID) and values of state variables (temperature, stress, etc.) at locations of interest.

As discussed previously, AVS is started and then connected to a Unix socket to accommodate bidirectional communication between AVS and the vetomain program. This allows vetomain to control the AVS process by issuing CLI (Command Line Interpreter) commands and also by receiving information about the model (such as node $\mathrm{ID}$ ) from AVS. Via this mechanism, the FEAVR environment is initialized within AVS.

There were two fundamental extensions to FEAVR that were necessary for VETO. One was the ability to "attach" a virtual instrument to the model at a user-selected node point (via a mouse click) with a user specified orientation. For example, as an analyst or test engineer reviews the mode shape shown in Figure 6, a virtual accelerometer can be placed at node 468 oriented parallel to the $Z$ axis. The location and orientation of the virtual instrument is then transferred back to vetomain for development of the model of the DUT needed in the time integration.

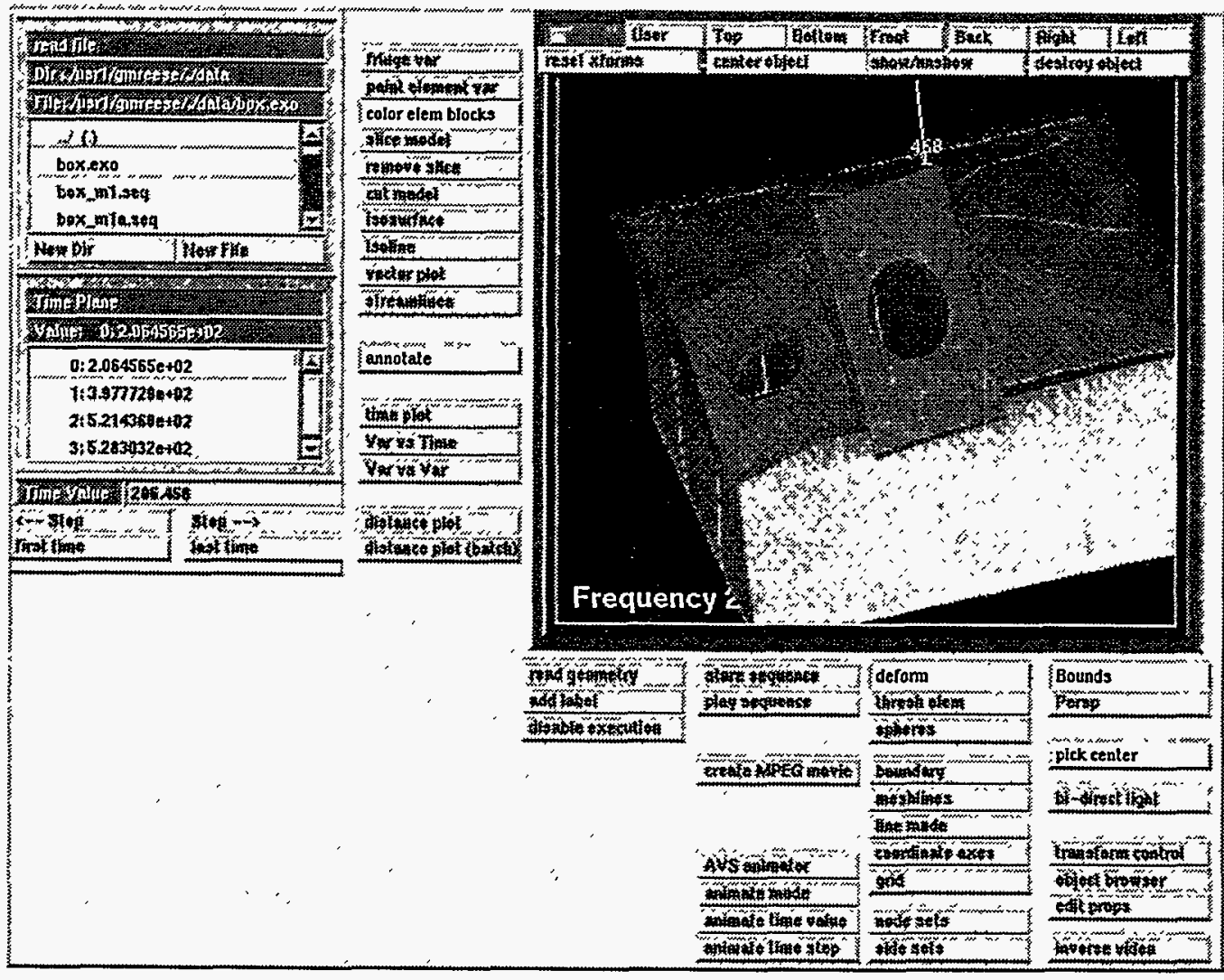

Figure 6. FEAVR Display. (Note virtual instrument at node 468.) 
The second extension to FEAVR was the capability to create "trace links," which are lines linking the virtual instruments on the model to create a simplified representation of the device geometry in the absence of the FE model. These are used in visualizing the simulated (or experimental) output of the virtual (or real) instruments. Figure 7 shows a deformed FE model (top) and the same model represented with trace links and virtual instruments.
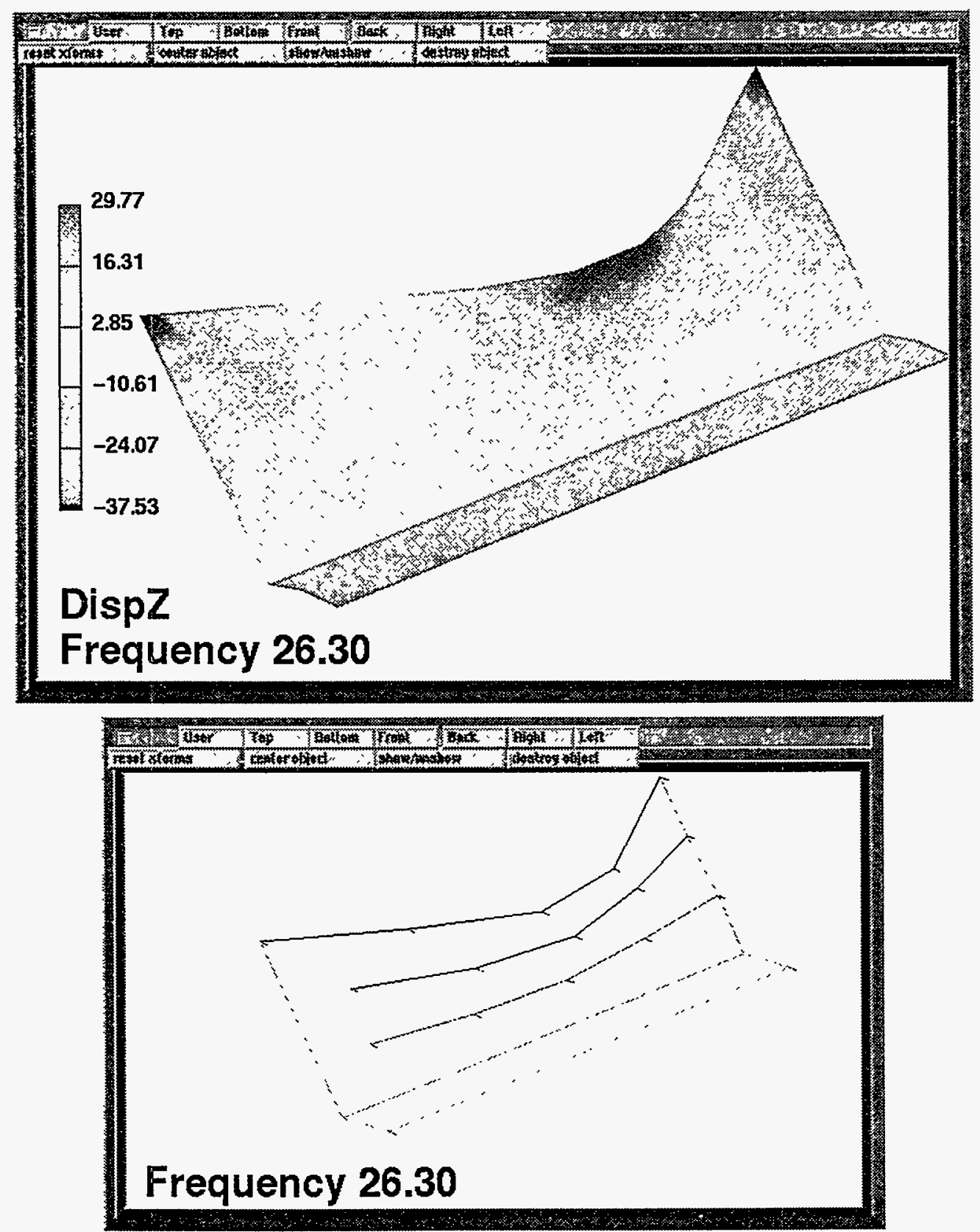

Figure 7. Deformed FE Model and Trace Lines From FEAVR 


\section{Simulations with MATLAB and SIMULINK}

The VETO software tool simulates the dynamic response behavior of a user-defined test environment. The SIMULINK Dynamic System Simulation Software toolkit provided by MATLAB is used as the environment to assemble and ultimately integrate mathematical models of the test system. This same toolkit controls the simulation processing. Dynamic response equations are integrated by SIMULINK to provide system output time histories. Within the VETO software, inputs such as type of device and interconnection of instrumentation models are combined to facilitate the rapid connection of various models (including models of test instrumentation, equipment and hardware) that comprise a given testing process. In order to achieve rapid set up of this virtual environment, models representing the instrumentation and test equipment need to be developed. These models consist of a mathematical description of the dynamic response of the instruments derived either theoretically or experimentally. Most of the instruments modeled to date have been modeled in the discrete state space domain. A number of system identification tools, e.g. Power Polynomial [5] and Eigensystem Realization Algorithm with Data Correlation II [6], were used in MATLAB to generate the mathematical models. Development was based on an experimental frequency response function of the instrument or equipment.

The models of the different types of instruments and equipment (transducers, amplifiers, filters, etc.) needed to represent a complete testing environment are located in a SIMULINK Virtual Test Equipment Library (VTELib). When preparing for a test simulation, the selection of the desired test instrumentation from the vetomain is performed with the assistance of a MATLAB M-file, which searches the VTELib for available instrument models. Optimal experimental design and simulation of the complete test environment is further facilitated by VETO's ability to include models of external inputs and electronic instrumentation noise. In addition, complex instrumentation models, such as the Front End data acquisition system, are constructed by combining multiple submodels to simulate the dynamic response behavior of the hardware.

When "Build Simulation" is chosen from the "Simulate" menu of vetomain, the analysis data describing the DUT and other selected instrumentation parameters are downloaded to the MATLAB workspace. Processing control is then passed to MATLAB to construct a SIMULINK model of the test system. Construction begins with a SIMULINK "new_system" operation specifying the user's selected name for the test system. The procedure places the device model blocks into this new system diagram specified by the vetomain data. There is a second level of block placement performed in the building process specific to the data acquisition device called the Front End. When the Front End model block is placed into the new system, additional submodel blocks that simulate AC coupling and anti-alias filtering are placed within the Front End block, based on the desired number of data simulation channels. Figure 8 shows a partial Front End block diagram as constructed by the VETO software tool. 


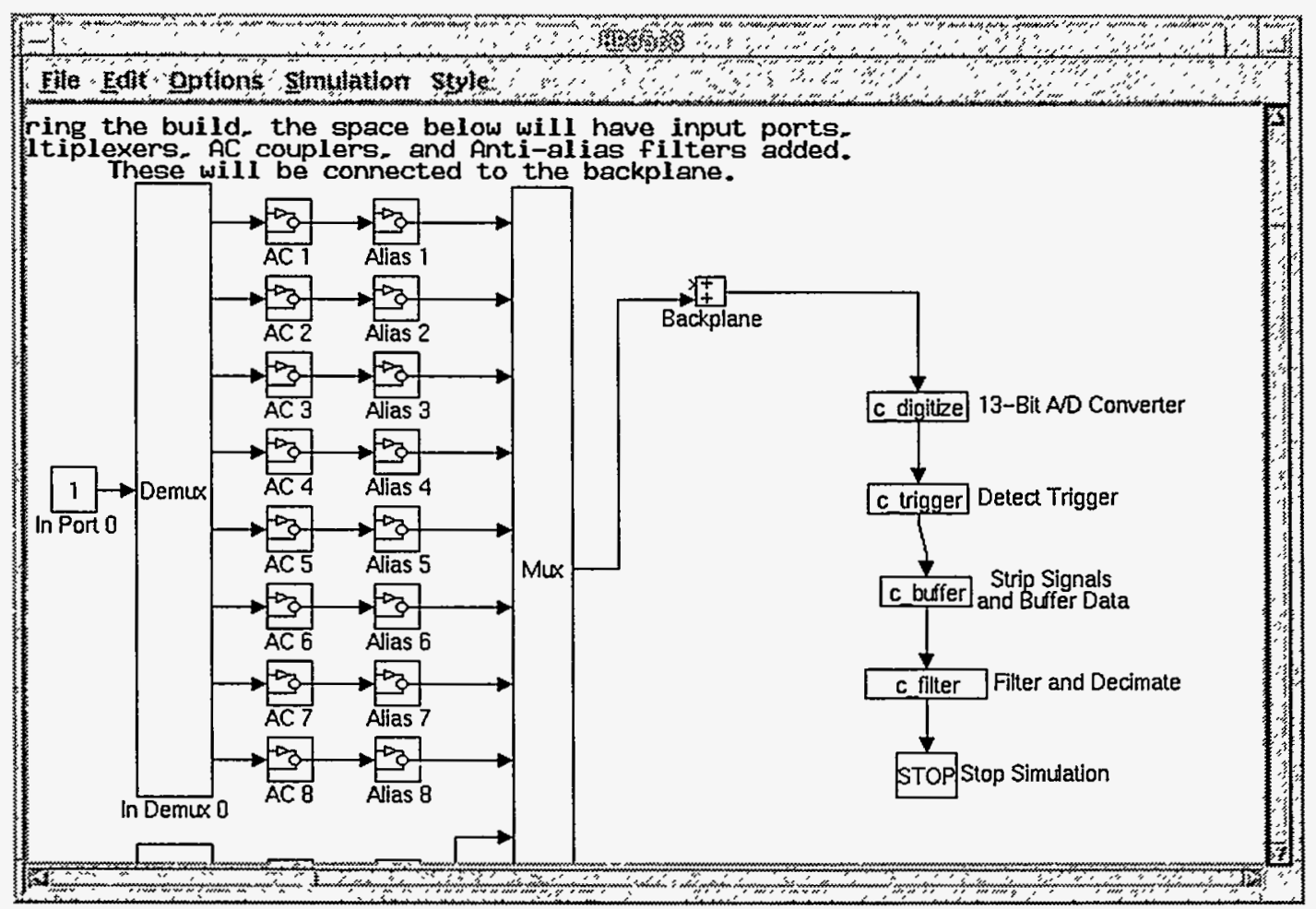

Figure 8. Partial Front End Block Diagram

As device blocks are added to the new system, interconnecting lines are placed between the blocks. These lines represent the flow of signals in the actual test system and are specified using the "wire" instrument in vetomain. Using these interconnecting lines, the input signals from the actuator devices (e.g. impact hammers) are fed to both the DUT for simulation of system excitation and to the Front End device for simulation of data acquisition. The Front End device also receives the signals from the sensors that have been attached to the DUT to simulate structural system response to the actuator input. Both the simulated actuator and sensor signals are linked through amplifier and filtering blocks to represent preconditioning of the signals.

The completed system is saved as a MATLAB ".m" and ".mat" file. Although it is possible for the user to modify the system built by VETO, care needs to be taken when directly modifying the SIMULINK simulation system. When changes are made to the test design from within SIMULINK, there is no mechanism for reflecting those changes in vetomain and in the FEAVR environment.

SIMULINK provides a number of methods for solving the set of differential equations that defines the mathematical model of the test system constructed in the build phase of VETO. The VETO tool uses a Runge-Kutta fourth-order (rk45 operation) method to numerically integrate the equations for the test system. This method is considered to be a good general-purpose integrator applicable to a large range of problems. It is a variable step size method with step size adjusted continuously to meet a specified relative error criterion. However, the VETO overrides the variable step size character by providing equal minimum and maximum step sizes as options when the simulation begins. The selected step size is 
the reciprocal of $32768 \mathrm{~Hz}$, the maximum sampling rate of the HP3565 Front End device used for data acquisition and analysis. This forces SIMULINK to calculate the system responses at a constant or uniform time interval during the simulation process.

The process of simulation begins when the user selects "Run" from the "Simulate" option on vetomain. The data files that define the dynamics of the desired instrumentation are loaded into the test simulation system, and the Simulation Monitor is created and displayed. This monitor allows the user to observe the estimated system response based on the numerical integration. The Simulation Monitor represents the data acquisition environment commonly used to gather data in a physical test and is a graphical interface through which the user interacts with the test simulation system. The monitor has a set of buttons to control the progress of the simulation and several display areas to provide visual feedback to the user. Figure 9 shows the monitor in its initial state prior to auto-ranging. The VETO tool automatically performs auto-ranging to simulate the setting of Front End data acquisition voltage ranges on each analog-to-digital convertor required in the test simulation.

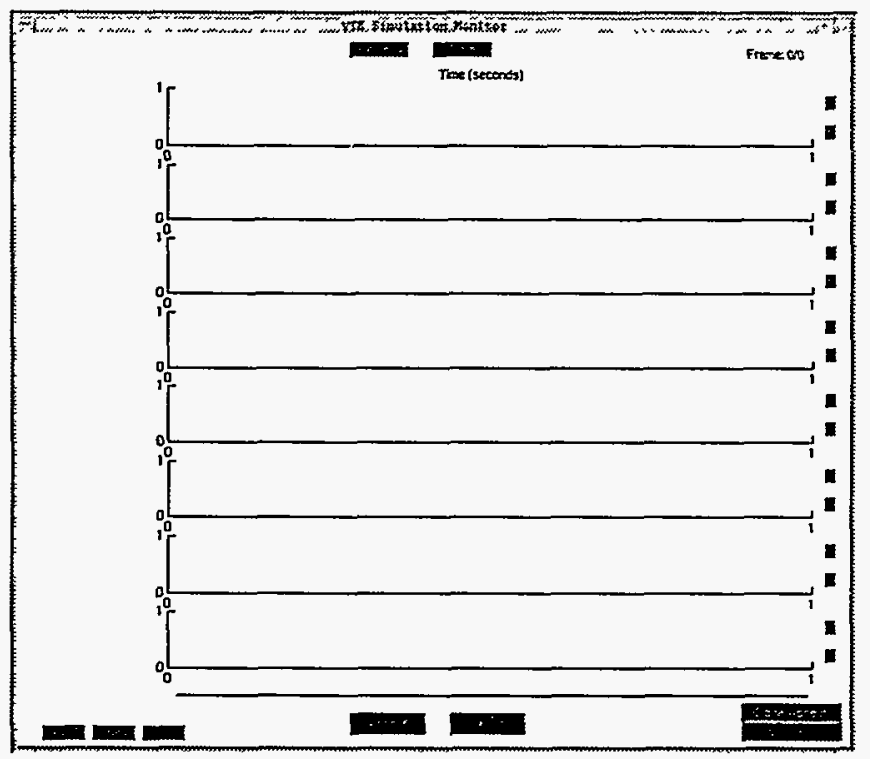

Figure 9. Simulation Monitor in Its Initial State

During the simulation, the user has the option to halt the run using a button on the monitor. Also, as each frame of data is collected, the simulated response is displayed on the monitor, and the user is provided visual feedback on the test simulation results. Voltage ranges for each channel can be varied in order to maximize the signal's dynamic range before performing post-simulation analysis. Each frame can be accepted or rejected as a valid set of data using control buttons found at the bottom of the monitor. A second set of buttons will accept or reject and also end the data collection phase. These buttons also activate a window providing an interface to analysis routines for computing desired measures such as frequency response functions, power spectral densities, and coherences. 


\section{Application of the VETO to Structural Dynamics Test Simulation}

The VETO software environment currently integrates analysis and test-based models to support optimal modal or structural dynamic test design. The structural dynamics testing environment was selected as the initial VETO environment for investigation into areas of design/analysis/test interfaces, visualization, versatility and repeatability. This initial VETO effort has focused on assisting engineers to maximize the value of modal tests. As was mentioned earlier, this environment plays a very critical role in the Knowledge Based Testing program, particularly for structural dynamics model validation problems.

A weapon component housing was selected as the test-case hardware for application in the VETO environment. The VETO software simulation tool was used to design an optimal experiment for the housing component. The goals of performing this test design optimization were to select an appropriate set of instrumentation (including sensors and actuators) to perform a modal experiment within the VETO environment, to simulate a modal test on the housing component, and then to compare the results of the simulation to actual experimental data. A finite element model of the housing structure was loaded into the VETO environment for use in the modal test simulation. The test design was performed over a frequency band which included the first six vibration modes of the housing structure. An experimental modal test was performed on the steel component housing, based on the VETO test design.

The outcome of the VETO test design "Setup" was to excite the structure at a single location using an impact hammer and to measure 51 acceleration responses on the housing component to characterize the dynamic behavior of the component (Figure 10). Small accelerometers, Endevco 2250s, were selected in the test design in order to minimize the mass loading effects during the experimentation. A large number of accelerometer locations were selected in the test design to make the process of analytical/experimental mode comparison more feasible. Other instrumentation such as the signal conditioning amplifiers and the Front End data acquisition system were also set up with the use of vetomain in preparation for the test simulation. Data acquisition parameters for sampling, averaging, and acquiring the desired analysis measurements were also selected for use in the post-simulation analysis.

A number of Pre-simulation tools were used to determine the completeness of the test design. First, the effects of mass loading the component housing were calculated given the test design sensor set (Figure 11). This figure shows that small changes in the frequencies of vibration would be experienced during the experimental test, based on the number of Endevco 2250 accelerometers chosen in the test design. Second, a normal mode indicator function and a driving point frequency response function were viewed before conducting the test simulation in order to assess whether the selected sensor and actuator (selected impact location) set would accurately identify all the desired modes of interest on the component housing (Figures 12 and 13). Initially, a location near the center of the housing, on the top of the dome, was selected for the excitation of the structure. By using the normal mode indicator function, it was determined that an input location at the edge of the housing would excite the first two modes of the structure more strongly than exciting at the center of the housing. Finally, the MAC was calculated for the test design to determine if the modes of vibration of the structure could easily be distinguished from one another given 
the selected sensor set. Small values on the off-diagonal terms of this MAC matrix, Figure 14 , indicate the relative independence of the modes of vibration, thus facilitating correlation with analysis.

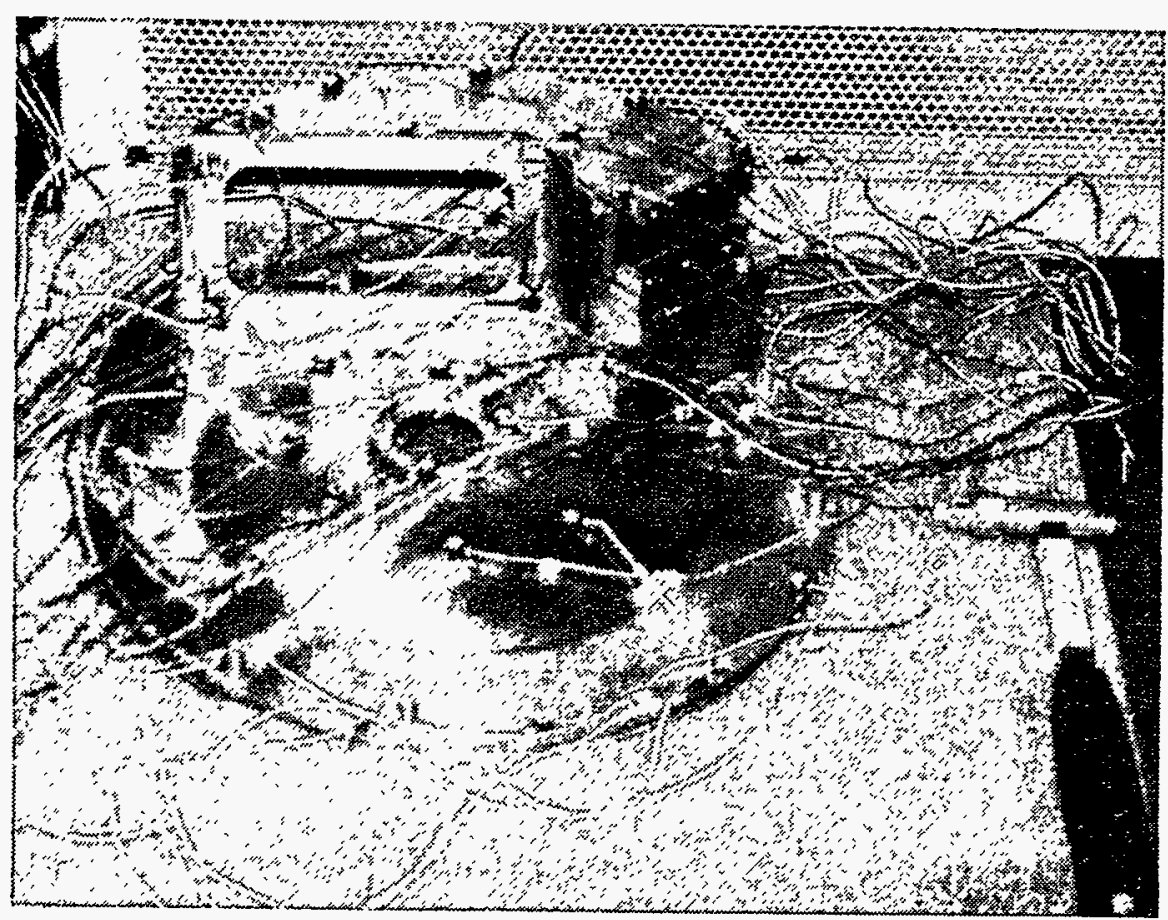

Figure 10. VETO Test Design for the Housing

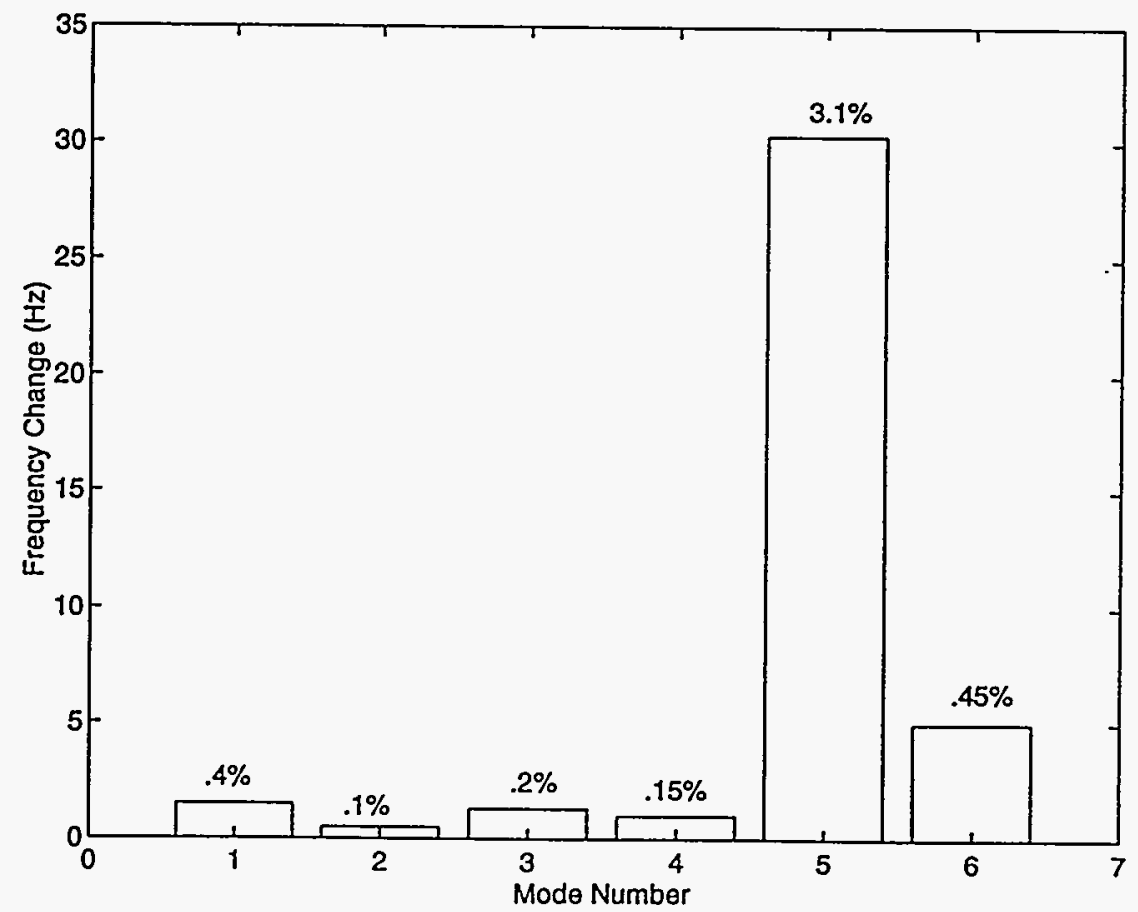

Figure 11. Estimated Frequency Shifts Due to Mass Loading the Housing. 


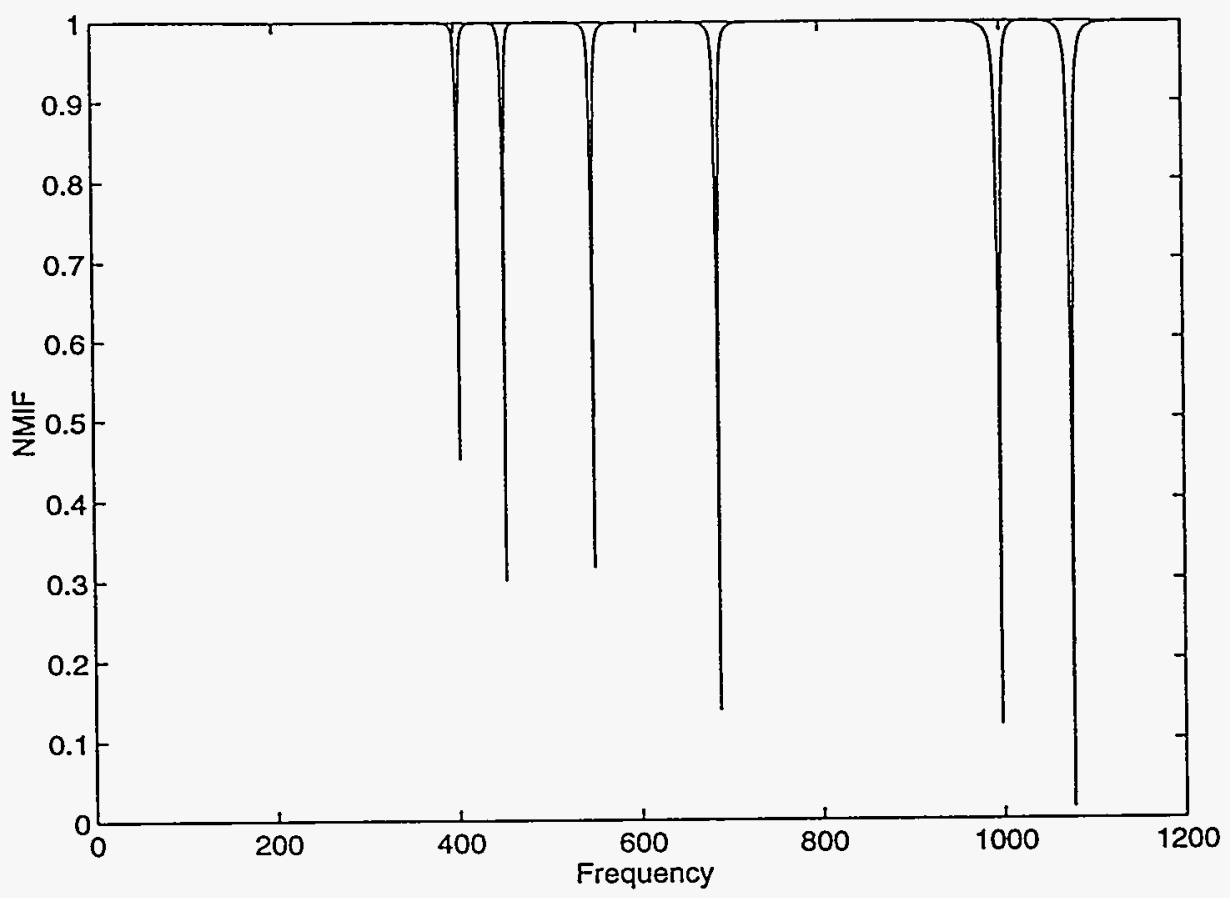

Figure 12. Normal Mode Indicator Function for the Housing
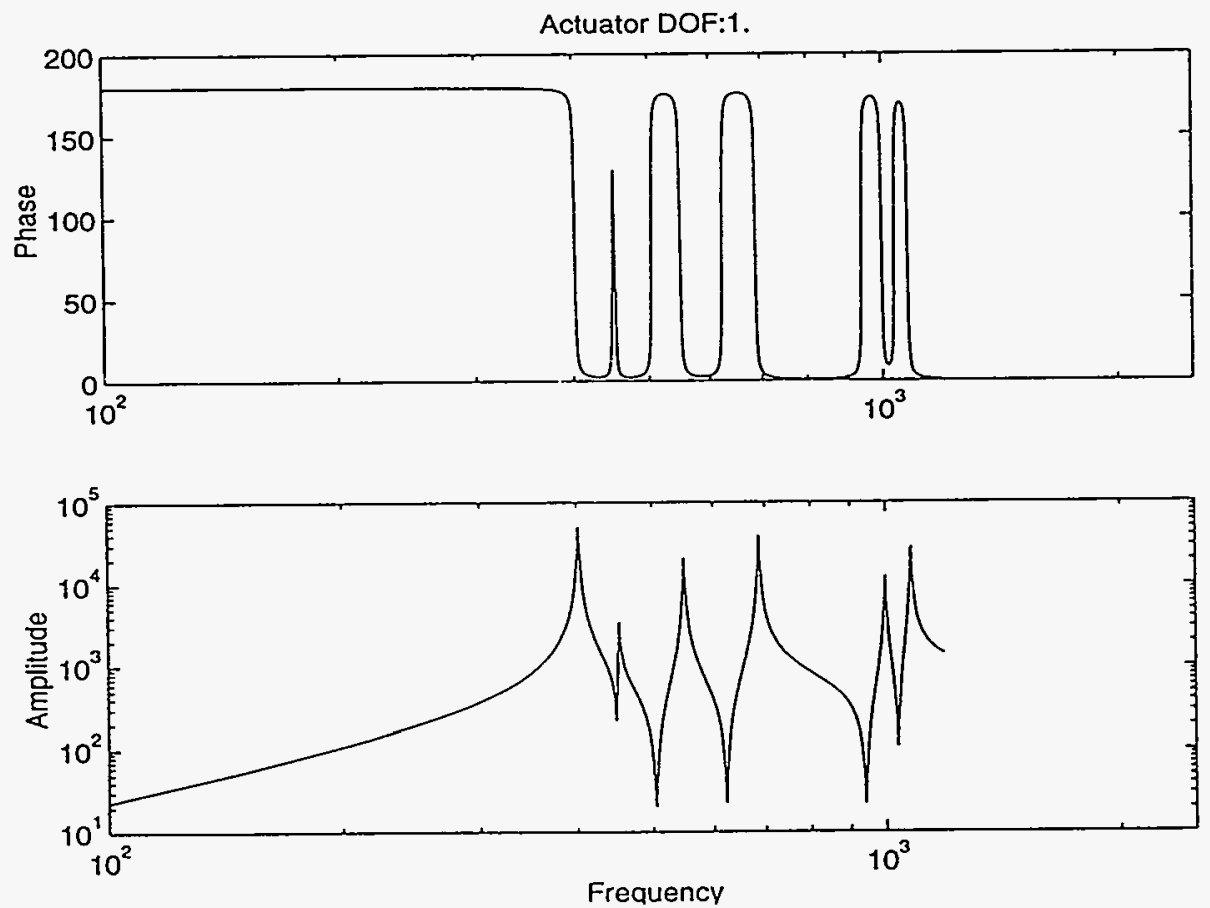

Figure 13. Driving Point Frequency Response for Housing 


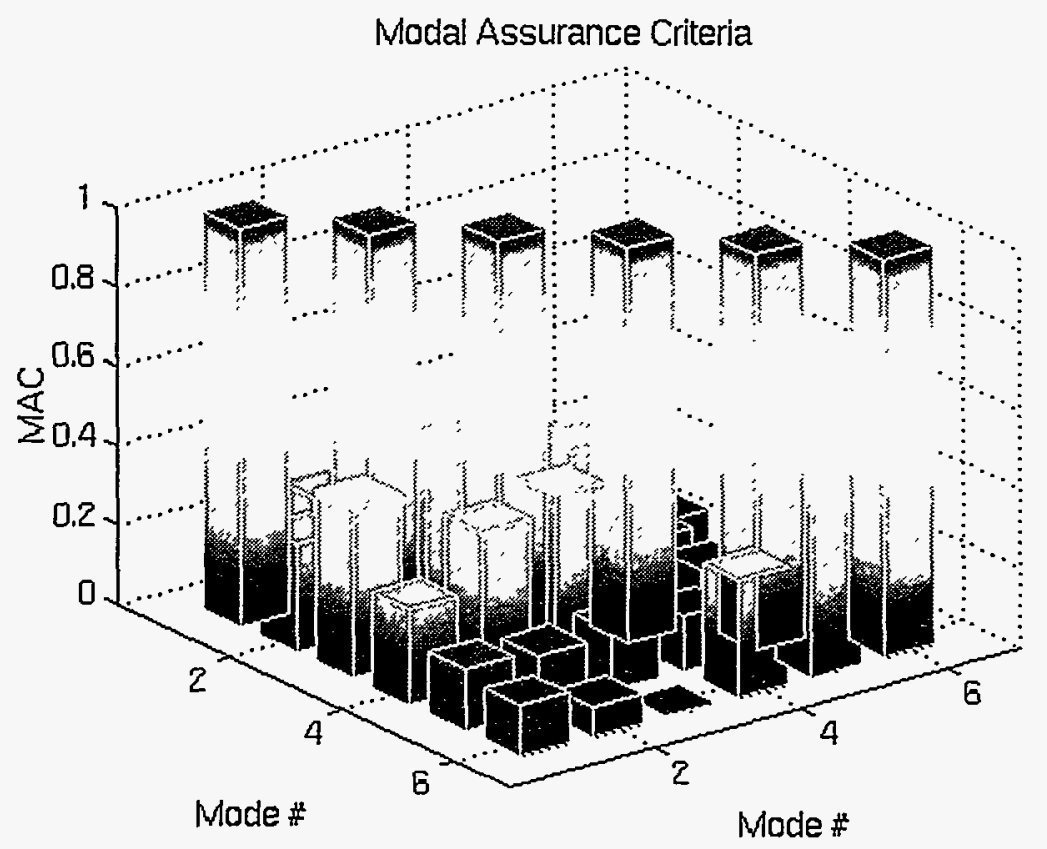

Figure 14. Modal Assurance Criterion (MAC) of the Housing

Once the test design had been completed within the VETO environment, a SIMULINK block model of the test environment was automatically generated to support the simulation of the modal test. Figure 15 shows a partial block model of this SIMULINK environment. The next step in the modal test simulation is the numerical integration of the mathematical models within SIMULINK to estimate the 51 system responses. Using the Simulation Monitor, these responses are observed for each set or frame of data to be collected (Figure 16). Once the data are gathered to support the desired measurement set, the test simulation within SIMULINK is concluded. A window that provides an interface to the post-simulation analysis routines is then used to download the data for measurement analysis. A comparison of frequency response functions for the simulated data and the experimental data for this test case is shown in Figure 17. The results of the comparison between the simulated and the actual experimental data show the need for computational model validation. The simulated data, which are based on the FE dynamic analysis, reflects lower modal frequencies than the experimental results. By using this experimental data in conjunction with the FE model, the computational model can be updated and used as a predictive tool. However, even with an inaccurate finite element model, the test was complete and well designed. Placement of sensors and actuators, as specified in the VETO, resulted in data from which the required modes could be extracted unambiguously. 


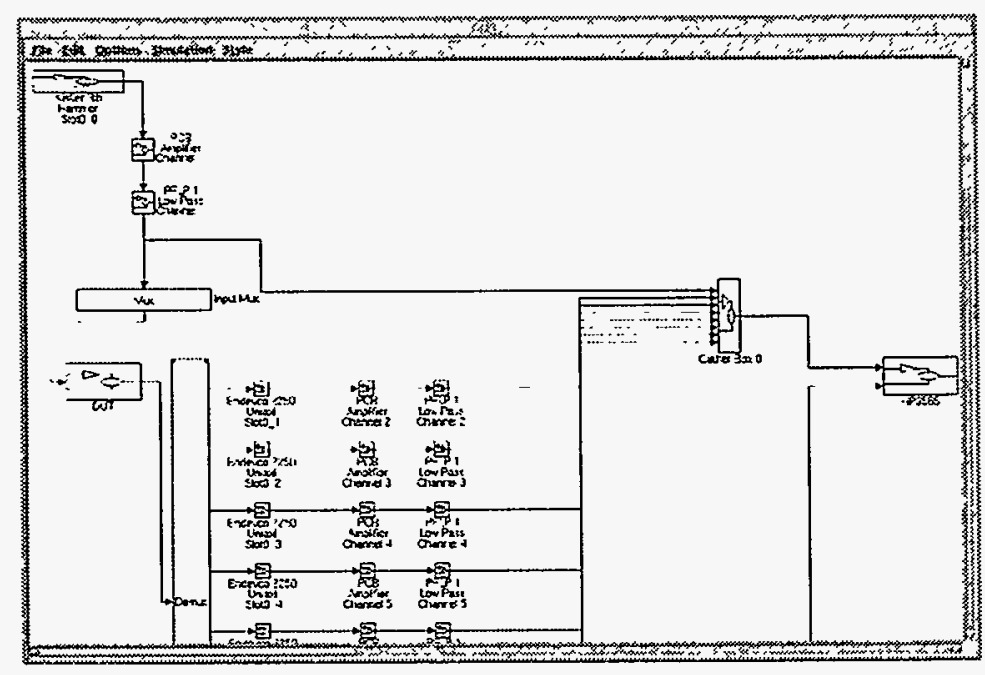

Figure 15. Partial Block Model of SIMULINK Environment

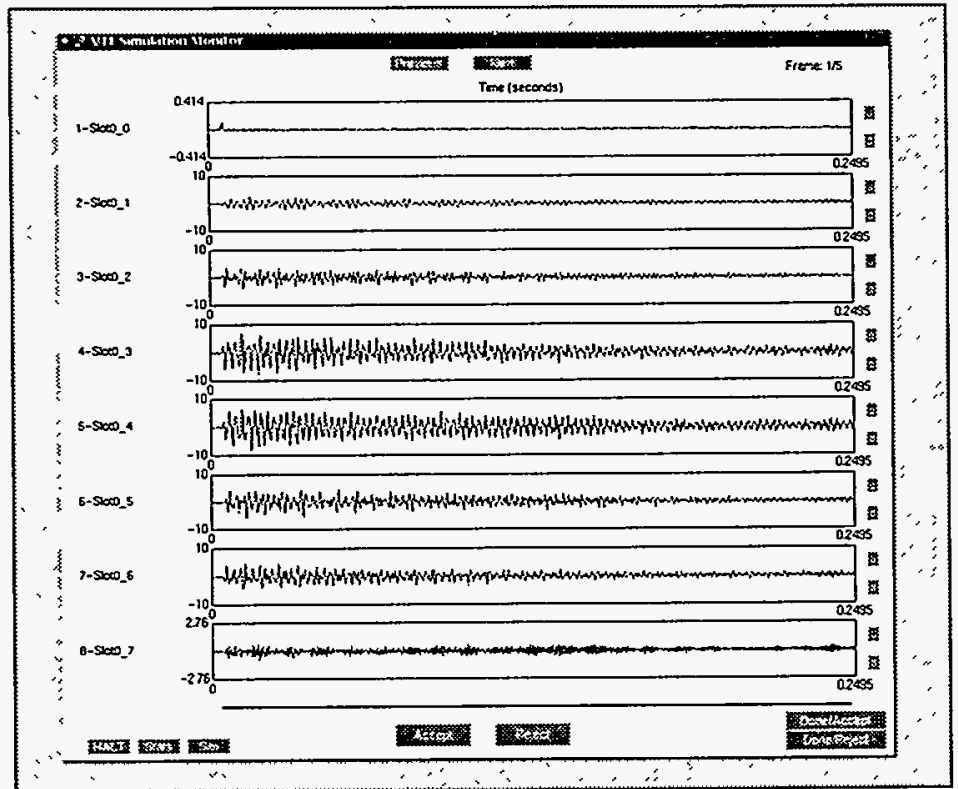

Figure 16. Collected Responses From Simulation Monitor 


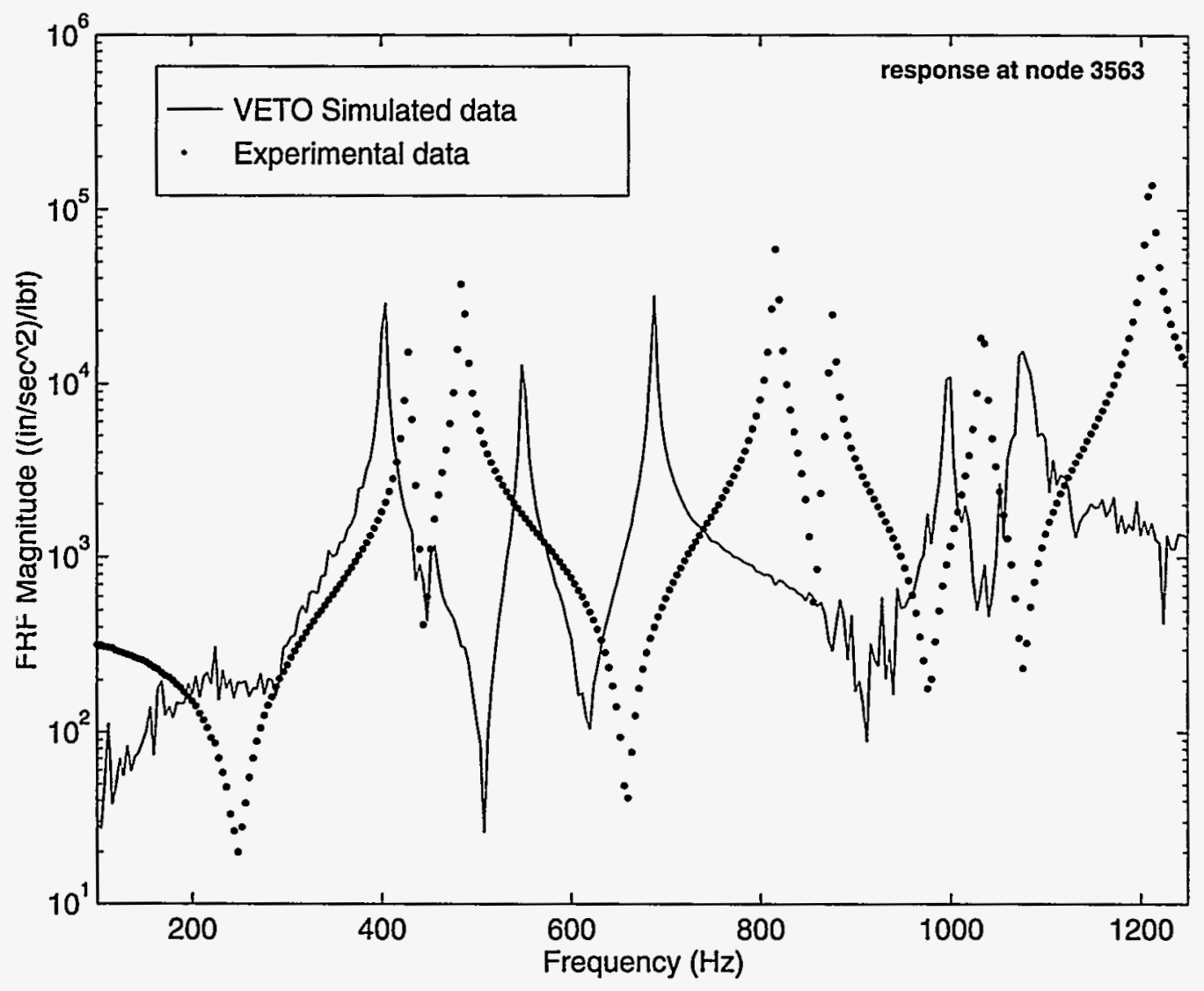

Figure 17. Comparison of Simulated and Experimental Frequency Response Functions 


\section{Conclusions}

The results of this weapon housing modal test design using the VETO environment clearly show the benefit of this software tool. Within this software environment, engineers were able to investigate the testing of this component prior to the existence of any hardware. The effects that different instrumentation or equipment had on the results of the experiment were observed, and the selection of appropriate analysis parameters were also studied. This tool assisted the engineers in the selection, placement and orientation of the instrumentation to maximize the information to be gathered from the experiment. Also, this tool allowed the visualization of results while iterating the test setup before committing to the actual test series. This test simulation tool, as previously described, plays an important role in the design of experiments for the purpose of computational model validation.

\section{References}

[1] L. A. Schoof, "Finite Element Analysis VieweR (FEAVR)," unpublished.

[2] L. A. Schoof and V. R. Yarberry, "EXODUS II: A Finite Element Data Model," Sandia Report SAND92-2137, September 1994.

[3] T. G. Carne and C. R. Dohrman, "A Modal Test Design Strategy for Model Correlation," Proceedings of the 13th International Modal Analysis Conference, 1995.

[4] D. C. Kammer, "Sensor Placements for On-Orbit Modal Identification and Correlation of Large Space Structures," Journal of Guidance, Control, and Dynamics, V14(2), 1991.

[5] P. S. Barney, "Power Polynomial Analysis Code," unpublished.

[6] J. P. Lauffer, "Eigensystem Realization Algorithm with Data Correlation II," unpublished. 


\section{DISTRIBUTION:}

5 Virginia Polytechnic Institute and State University

Attn: R. L. West

Mechanical Engineering Dept.

Blacksburg, VA 24061-0238

\begin{tabular}{|c|c|c|}
\hline 1 & MS 9405 & D. L. Lindner, 1809 \\
\hline 1 & 0429 & R. D. Andreas, 2100 \\
\hline 1 & 0445 & R. C. Hartwig, 2166 \\
\hline 1 & 0481 & R. G. Bell, 2167 \\
\hline 1 & 9005 & J. B. Wright, 2200 \\
\hline 1 & 0509 & W. D. Williams, 2300 \\
\hline 1 & 0505 & D. L. Eilers, 2336 \\
\hline 1 & 0859 & S. M. Gutierrez, 2527 \\
\hline 1 & 0458 & R. K. Thomas, 5100 \\
\hline 1 & 0458 & J. S. Rottler, 5139 \\
\hline 1 & 9405 & C. F. Acken, 8220 \\
\hline 1 & 9007 & R. C. Wayne, 8400 \\
\hline 1 & 0151 & G. Yonas, 9000 \\
\hline 1 & 0841 & P. J. Hommert, 9100 \\
\hline 1 & 0835 & R. D. Skocypec, 9102 \\
\hline 1 & 0833 & J. H. Biffle, 9103 \\
\hline 1 & 0826 & M. W. Glass, 9111 \\
\hline 1 & 0443 & S. N. Burchett, 9117 \\
\hline 1 & 0443 & H. S. Morgan, 9117 \\
\hline 1 & 0437 & C. R. Adams, 9118 \\
\hline 1 & 0437 & E. P. Chen, 9118 \\
\hline 1 & 0321 & E. H. Barsis, 9200 \\
\hline 1 & 0318 & G. S. Davidson, 9215 \\
\hline 5 & 0441 & L. A. Schoof, 9225 \\
\hline 6 & 0441 & P. L. Stanton, 9225 and staff \\
\hline 1 & 0819 & J. M. McGlaun, 9231 \\
\hline 1 & 0820 & P. Yarrington, 9232 \\
\hline 18 & 0439 & D. R. Martinez, 9234 and staff \\
\hline 5 & 0439 & G. M. Reese, 9234 \\
\hline 1 & 0949 & R. W. Harrigan, 9602 \\
\hline 1 & 1006 & P. Garcia, 9671 \\
\hline 1 & 0507 & K. G. McCaughey, 9700 \\
\hline 1 & 0555 & R. A. May, 9706 \\
\hline 1 & 0555 & D. B. Davis, 9732 \\
\hline 1 & 0557 & D. O. Smallwood, 9735 \\
\hline 1 & 0865 & J. L. Moya, 9735 \\
\hline 1 & 0557 & W. N. Dunn, 9735 \\
\hline 1 & 0557 & J. S. Cap, 9735 \\
\hline 20 & 0557 & T. J. Baca, 9741 and staff \\
\hline 3 & 0557 & S. E. Klenke, 9741 \\
\hline
\end{tabular}




\section{DISTRIBUTION (concluded):}

$\begin{array}{lll}1 & 0555 & \text { M. S. Garrett, } 9742 \\ 1 & 0555 & \text { D. L. Gregory, } 9742 \\ 1 & 0555 & \text { B. D. Hansche, 9742 } \\ 1 & 0555 & \text { T. C. Togami, } 9742 \\ 1 & 0615 & \text { W. W. Scurtleff, 9752 } \\ 1 & 1135 & \text { J. R. Garcia, 9761 } \\ 1 & 0163 & \text { J. Polito, } 9800 \\ 1 & 0163 & \text { D. M. Rondeau, 9803 } \\ 1 & 9018 & \text { Central Technical Files, 8523-2 } \\ 5 & 0899 & \text { Technical Library, 4414 } \\ 1 & 0619 & \text { Print Media, 12615 } \\ 2 & 0100 & \text { Document Processing, 7613-2 } \\ & & \text { For DOE/OSTI }\end{array}$

